\title{
Multisite comparison of wheelchair propulsion kinetics in persons with paraplegia
}

Alicia M. Koontz, PhD, RET; ${ }^{1-2 *}$ Yusheng Yang, PhD; ${ }^{\mathbf{1}}$ Robert Price, MSME; ${ }^{\mathbf{3}}$ Michelle L. Tolerico, MS; $\mathbf{1}^{\mathbf{1 , 4}}$
Carmen P. DiGiovine, PhD, RET; Sue Ann Sisto, PhD; ${ }^{6-7}$ Rory A. Cooper, PhD; ${ }^{1-2}$ Michael L. Boninger, MD; ${ }^{\mathbf{1}-\mathbf{4}}$
${ }^{1}$ Human Engineering Research Laboratories, Department of Veterans Affairs Pittsburgh Healthcare System,
Pittsburgh, PA; ${ }^{2}$ Departments of Rehabilitation Science and Technology and Bioengineering, University of Pittsburgh,
Pittsburgh, PA; ${ }^{3}$ Department of Rehabilitation Medicine, University of Washington, Seattle, WA; ${ }^{4}$ Department of Phys-
ical Medicine and Rehabilitation, University of Pittsburgh Medical Center Health System, Pittsburgh, PA; ${ }^{5}$ Assistive
Technology Unit, Department of Disability and Human Development, University of Illinois at Chicago, Chicago, IL;
${ }^{6}$ Department of Physical Medicine and Rehabilitation, University of Medicine and Dentistry of New Jersey-New Jersey
Medical School, Newark, NJ; ${ }^{7}$ Kessler Medical Rehabilitation Research and Education Corporation, West Orange, NJ

Abstract-A multisite collaborative study is being conducted on the association between propulsion biomechanics and upperlimb injuries. This substudy compared subject characteristics and pushrim kinetics across three sites and identified early on in the main study any differences that could affect interpretation of the findings or data pooling. A total of 42 manual wheelchair users with paraplegia (14 from each site) performed $0.9 \mathrm{~m} / \mathrm{s}$ and $1.8 \mathrm{~m} / \mathrm{s}$ steady state propulsion trials and an acceleration-brakecoastdown trial on a wheelchair dynamometer while propulsion forces and moment about the hub were measured with a SmartWheel. Significant differences between two sites were found in peak and average resultant force $(p<0.05)$, peak and average moment at the slower steady state speed $(p<0.005)$, and peak and average torque at the faster steady state speed $(p=0.06)$. Subjects at the site with significantly lower forces and torques had a slower deceleration rate during coastdown compared with the subjects at the other two sites $(p<0.001)$. These results imply that rolling resistance is lower at one of the sites and likely due to differences in dynamometer properties. A mechanical method was used to site-normalize the data and enable data pooling for future analyses.

Key words: biomechanics, friction, inertia, kinetics, manual wheelchair, paraplegia, propulsion, rehabilitation, rolling resistance, spinal cord injury, wheelchair dynamometer.

\section{INTRODUCTION}

Shoulder and wrist pain is highly prevalent among persons with spinal cord injury (SCI) who use manual wheelchairs [1]. Performing routine activities such as transfers, push-ups for weight relief, and wheelchair propulsion are believed to be responsible for the development of upper-limb pain and repetitive strain injuries [24]. Prior studies have found a direct causal link between wheelchair propulsion biomechanics and injuries [5-8].

\footnotetext{
Abbreviations: $3-\mathrm{D}=$ three-dimensional, $3 \mathrm{MP}=$ third metacarpalphalangeal joint, ANCOVA = analysis of covariance, ANOVA = analysis of variance, HERL = Human Engineering Research Laboratories, KMRREC = Kessler Medical Rehabilitation Research and Education Corporation, NIDRR = National Institute on Disability and Rehabilitation Research, SCI = spinal cord injury, $\mathrm{SD}=$ standard deviation, $\mathrm{T}=$ thoracic, $\mathrm{UW}=\mathrm{Uni}-$ versity of Washington.

*Address all correspondence to Alicia M. Koontz, PhD, RET; Human Engineering Research Laboratories, Department of Veterans Affairs Pittsburgh Healthcare System, 7180 Highland Drive, 151R1-H, Pittsburgh, PA 15206; 412365-4850; fax: 412-365-4858. Email: akoontz@pitt.edu DOI: 10.1682/JRRD.2006.05.0048
} 
These studies, like other wheelchair propulsion studies in general [9-12], had small sample sizes and lacked the statistical power necessary to model the complex interactions among subject demographics, wheelchair characteristics, propulsion biomechanics, and injury. To address this limitation, a multisite, multiagency study is investigating these interactions in a large group of manual wheelchair users from three different geographical locations. The sites involved in the study are the Human Engineering Research Laboratories (HERL) in Pittsburgh, Pennsylvania (coordinating site), the Kessler Medical Rehabilitation Research and Education Corporation (KMRREC) in Orange, New Jersey, and the University of Washington (UW) in Seattle, Washington. The commonality that drew these three sites together was their involvement in the SCI Model Systems Program funded by the National Institute on Disability and Rehabilitation Research.

As the coordinating site, HERL worked with the other two sites to develop uniform procedures for measuring propulsion biomechanics. All sites purchased a SmartWheel ${ }^{\circledR}$ (Three Rivers Holding, Mesa, Arizona) for measuring the three-dimensional (3-D) forces and torques at the pushrim. Each site had an existing 3-D motion capture system that could be dedicated to data collection. In addition, HERL designed and manufactured three wheelchair dynamometers, one for each site. The study protocol was modeled after prior HERL studies [7].

During the first year of the study, a procedures manual was developed with specific details and instructions concerning experimental setup (e.g., securing wheelchair to dynamometer, SmartWheel setup, kinematic marker placement), data collection methods (e.g., number and type of propulsion trials, length of data collection), equipment maintenance and calibrations, data checking, transfer, and processing procedures. This manual was created so that each site followed the same standardized procedures when testing each subject. A secure Web site was created so that all sites could have immediate access to the manual during testing. All investigators have met three times in person, one time at each site, to review the protocol and procedures manual and to perform mock trials. All investigators participate in a conference call every 2 weeks to discuss study-related issues (e.g., protocol addendums, recruitment, equipment) and progress.

The multisite, multiagency study is currently in the data collection phase. This substudy compared subject characteristics and pushrim kinetics across sites to assess uniformity and identify early on in the main study any differences that could affect interpretation of the findings or data pooling. If differences were found, we intended to develop a method to correct them and unify the sites' data. The outcomes of the multisite, multiagency study could lead to greater understanding of the subject and biomechanical characteristics that contribute to the development of upper-limb injuries among individuals with paraplegia.

\section{METHODS}

The Pittsburgh Department of Veterans Affairs Research and Development Human Studies Subcommittee, the University of Pittsburgh Institutional Review Board, the UW Human Subjects Division, and the KMRREC Institutional Review Board approved this study.

\section{Subjects}

A total of 42 subjects, 14 from each site, volunteered for the study and provided informed consent. All subjects used a manual wheelchair as their primary means of mobility and had an SCI below thoracic (T) 1 that had occurred more than 1 year before the start of the study. Subjects also had wheelchairs with quick-release wheels to ensure compatibility with the kinetic measurement device.

\section{Equipment}

Each site used the SmartWheel, a 3-D force- and torque-sensing pushrim (Three Rivers Holdings, Inc, Mesa, Arizona), to collect data on propulsion kinetics. The SmartWheel has demonstrated excellent accuracy, linearity, and precision [13-14]. The manufacturer initially calibrates the SmartWheel. The calibration process results in a set of constants that are used to convert the raw digital voltage readings to each respective force $\left(F_{x}, F_{y}, F_{z}\right)$ and torque $\left(M_{x}, M_{y}, M_{z}\right)$ component. Every 6 months, each site performs a "calibration check" by hanging known weights from the wheel in various positions and verifying the measured output and constants.

Kinematic measurements were obtained with 3-D motion capture systems. Each site used a different system, but all were capable of outputting 3-D marker position data relative to a global origin (located between the two rollers of the wheelchair dynamometer). The UW site used a Qualisys MCU240 (Qualisys AB, Gothenburg, Sweden), the KMRREC site used a VICON 612 Workstation (Vicon 
Peak, Lake Forest, California), and the HERL site used two Optotrak 3020 systems (Northern Digital, Inc, Waterloo, Ontario, Canada). A pendulum test is performed every 6 months to provide a basis of comparison of kinematic data collection systems between sites. Three markers, separated by a prescribed fixed distance, are placed on the arm of a pendulum that is put into motion within the calibrated volume of the kinematic motion-capture system. The intermarker separation is computed over a capture period to ensure stability of the marker separation.

Three wheelchair dynamometers were fabricated and assembled at HERL, and one was installed at each participating site. They were modeled after the dynamometer described in DiGiovine et al. [15], without the computercontrolled motors and torque sensors. The dynamometer consists of two independent steel tubular rollers, one for each wheel, supported by pillow-block bearings. The bearings are mounted to steel channels. Attached to each channel and roller is a tachometer (SB740A-7, ServoTek, Hawthorne, New Jersey) that provides velocity feedback to the wheelchair user while he or she propels on the dynamometer. Signals from both tachometers are read into an A/D (analog/digital) data acquisition board and a custom LabVIEW (National Instruments, Inc, Austin, Texas) program displays the average roller speed. The program also has an indicator (a needle that moves left and right depending on individual roller speed) to guide the subject in pushing at the same speed on both sides. Routine checks and maintenance on the dynamometer are performed every 6 months. Each site performs a coastdown test and records the time it takes for each roller to coast from $1 \mathrm{~m} / \mathrm{s}$ to rest. Since independent rollers are used, side-to-side differences in coastdown times are also assessed. Tachometer measures are cross-checked with the velocity of the SmartWheel every 6 months.

\section{Experimental Protocol}

The SmartWheel was installed on the subject's nondominant side and on his or her own wheelchair that was secured to the dynamometer as shown in the Figure. The nondominant side was studied since it is less likely to be injured from repetitive use other than wheelchair propulsion. A kinematic marker was placed on the third metacarpalphalangeal joint (3MP) and the hub of the wheelchair to determine the hand contact angle. The velocity feedback program was displayed on a computer screen in front of the subject. Once the SmartWheel was on and the wheelchair attached to the dynamometer, the subject was asked to push the chair to become acclimated to the dyna-

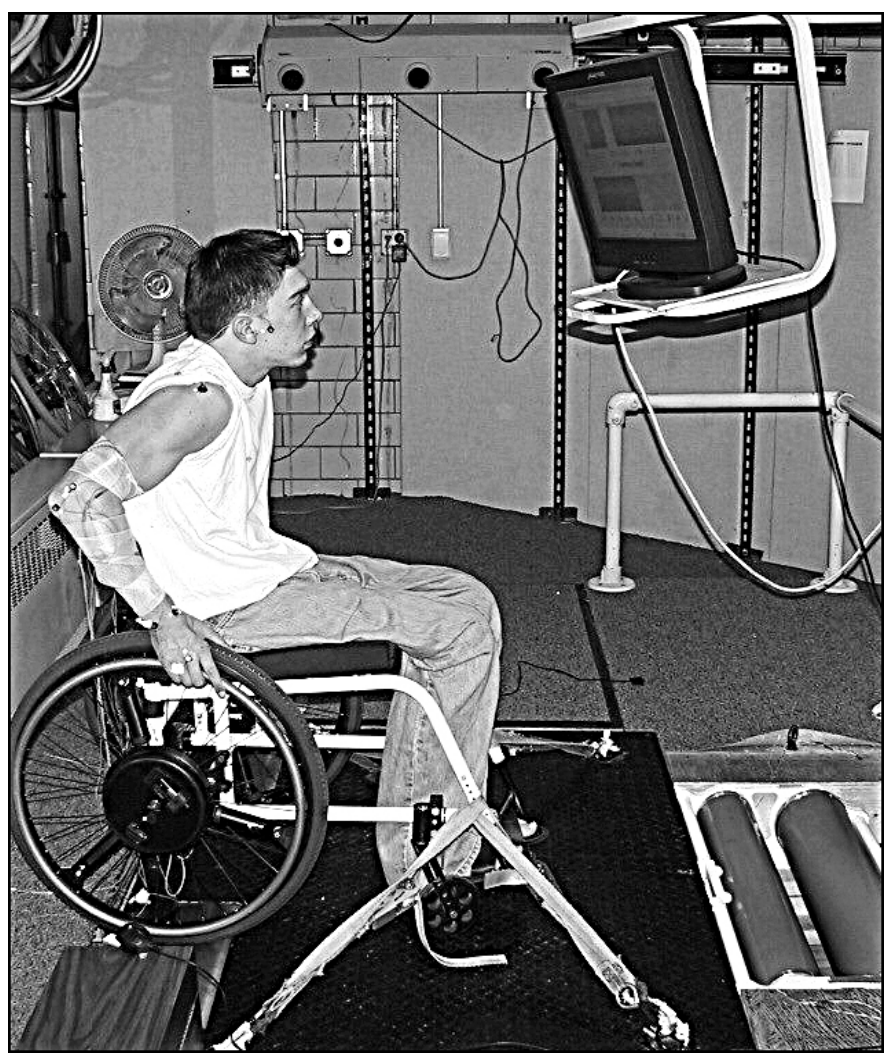

Figure.

Experimental setup at Human Engineering Research Laboratories.

mometer. Once comfortable, the subject performed two separate steady state speed propulsion trials at $0.9 \mathrm{~m} / \mathrm{s}$ and $1.8 \mathrm{~m} / \mathrm{s}$. When the subject reached the target speed, data collection was initiated and continued for $20 \mathrm{~s}$. In a third trial, subjects were instructed to start propelling from a resting position to their fastest possible speed, brake to an immediate stop, and push to fastest speed again until receiving a verbal cue to let go of the wheels and coastdown to a complete stop. Total data collection time for this trial was $30 \mathrm{~s}$. Rather than having scheduled rest breaks between trials, subjects were told that they could take a break whenever they felt tired.

\section{Data Analysis}

\section{Steady State Speed Trials}

We determined the beginning and end of each propulsion stroke by visually inspecting all three components of force and torque and determining the instant that all forces and moments deviated from baseline (start of the stroke) or approached baseline (end of the stroke). The resultant pushrim force from the SmartWheel was computed from 
the vector sum of the 3-D force components, $F_{x}, F_{y}$, and $F_{Z}$. Peak and average magnitudes of the resultant force $(F)$ and moment about the hub $\left(M_{z}\right)$ were determined for the first 10 strokes for each constant speed trial. In addition, contact angle (angular distance that forces were applied on the wheel for each stroke), average velocity per stroke, and the number of strokes per second were averaged for the same 10 strokes in each steady state trial. The 3MP marker of one of the UW subjects was indiscernible during the fast speed trial because of marker dropout. Therefore, the reported mean contact angles for the UW site at this speed are based on 13 subjects.

\section{Acceleration-Brake-Coastdown Trial}

Only the first stroke and coastdown portion of this trial were analyzed. The same parameters calculated for the steady state trials were also calculated for the first stroke. Coastdown was analyzed to determine whether the dynamometers were functioning similarly at each site. The starting point of coastdown was discerned by visual inspection of the $M_{z}$ curve. Where $M_{z}$ approached zero at the end of the last stroke in the trial marked the beginning of coastdown. From this point forward, the wheel angular position curve obtained from the SmartWheel optical encoder was converted to a linear position (distance) curve and curve-fitted to the second-order quadratic Equation

$$
d=\frac{1}{2} a t^{2}+v t+d_{0},
$$

where $d=$ distance traveled in meters, $t=$ time in seconds, $a=$ acceleration in meters per seconds squared (deceleration when constant is negative), $v=$ initial velocity in meters per second, and $d_{0}=$ initial distance in meters. At the start of coastdown, we initialized the distance to zero. The constants obtained from the curve-fit analysis yielded the subject's constant deceleration, initial velocity, and initial distance. Data for the coastdown portion for one subject at the UW site were incomplete (trial ended before the wheelchair came to a complete stop) and therefore were not included in the final analysis. For each coastdown trial, we also determined the time it took for each subject to coastdown from $1 \mathrm{~m} / \mathrm{s}$ to rest.

\section{Statistical Analysis}

Descriptive analysis, including mean and standard error for continuous variables and frequency for categorical variables, was initially performed to describe the subject demographics. One-way analysis of variance (ANOVA) was used for comparison of all subject demographic variables across the three sites except for injury level, which was converted to a numerical equivalent (e.g., $\mathrm{T} 2=2$, T3 $=3$, T4 = 4) and compared with use of a Kruskal-Wallis test. Sex was compared with the Fisher Exact test. The biomechanical data were compared across the sites with analysis of covariance (ANCOVA) models that controlled for sex, years postinjury, and body weight. Bonferroni pairwise comparisons were performed on significant variables. Coastdown model constants and times were compared with a one-way ANOVA test and post hoc Bonferroni test that adjusted the $p$-value to control for family-wise error ( $p=p$ unadjusted $\times$ number of tests). Pearson correlations ( $r$ ) were used to assess the relationship between body weight and coastdown deceleration. Data were postprocessed in MATLAB (MathWorks, Inc, Natick, Massachusetts) and statistically analyzed with Statistical Package for the Social Sciences 11.0 for Windows software package (SPSS Inc, Chicago, Illinois) and Statistical Analysis Software version 8 (SAS Institute, Inc, Cary, North Carolina). Verification of model assumptions and fit were carried out via examination of residual plots. The level of statistical significance was set at 0.05 for all statistical analyses. Trends of significance were noted when $p<0.10$.

\section{RESULTS}

\section{Subject Demographics}

Subject demographics are shown in Table 1. Subjects at HERL had had their SCI longer than subjects at KMRREC $(p=0.03)$ and UW $(p=0.08)$. Age, body weight, level of SCI, and sex were not statistically different across sites (Table 1).

\section{Steady State Speed Trials}

For the $0.9 \mathrm{~m} / \mathrm{s}$ speed trial, peak and average resultant force and wheel moment were significantly different between sites, with UW subjects pushing with less force and moment in comparison with HERL subjects (Table 2). Marginal significance was found between HERL and UW for the contact angle, with UW subjects propelling with smaller contact angles. For the $1.8 \mathrm{~m} / \mathrm{s}$ trial, a trend of significance was found for the peak and average wheel moment, with UW subjects pushing with less moment in comparison with HERL subjects (Table 3). All sites pushed with measured velocities close to the target velocities and with similar stroke frequencies. 
Table 1.

Subject demographics for three study sites: Kessler Medical Rehabilitation Research and Education Corporation (KMRREC), University of Washington (UW), and Human Engineering Research Laboratories (HERL). Data presented as mean \pm standard error, except injury level and sex.

\begin{tabular}{lcccc}
\hline \multicolumn{1}{c}{ Demographic } & $\begin{array}{c}\text { KMRREC } \\
(\boldsymbol{n}=\mathbf{1 4})\end{array}$ & $\begin{array}{c}\text { UW } \\
(\boldsymbol{n}=\mathbf{1 4})\end{array}$ & $\begin{array}{c}\text { HERL } \\
(\boldsymbol{n}=\mathbf{1 4})\end{array}$ & $\begin{array}{c}\text { All Sites } \\
(\boldsymbol{n}=\mathbf{4 2})\end{array}$ \\
\hline Age $(\mathrm{yr})$ & $37.8 \pm 3.86$ & $42.9 \pm 3.04$ & $43.2 \pm 2.67$ & $41.3 \pm 1.86$ \\
Weight $(\mathrm{kg})$ & $82.1 \pm 5.03$ & $72.6 \pm 3.58$ & $73.2 \pm 2.46$ & $76.0 \pm 2.26$ \\
Years Postinjury & $10.3 \pm 2.75$ & $11.5 \pm 1.92$ & $19.1 \pm 2.22^{*}$ & $13.6 \pm 1.44$ \\
Injury Level (median) & $9.5(\mathrm{~T} 9 / \mathrm{T} 10)$ & $8(\mathrm{~T} 8)$ & $7(\mathrm{~T})$ & $8(\mathrm{~T})$ \\
Sex, \% female $(n)$ & $7.1(1$ woman) & $42.9(6$ women) & $14.3(2$ women $)$ & $21.4(9$ women) \\
\hline
\end{tabular}

${ }^{*}$ HERL significantly different from KMRREC $(p=0.03)$ and marginally significantly different from UW $(p=0.08)$.

$\mathrm{T}=$ thoracic.

Table 2.

Constant speed trial at $0.9 \mathrm{~m} / \mathrm{s}$ : Temporal kinetic data (mean \pm standard error) for each site after controlling for sex, years postinjury, and weight.

\begin{tabular}{lccc}
\hline \multicolumn{1}{c}{ Variable } & $\begin{array}{c}\text { KMRREC } \\
(\boldsymbol{n}=\mathbf{1 4})\end{array}$ & $\begin{array}{c}\text { UW } \\
(\boldsymbol{n}=\mathbf{1 4})\end{array}$ & $\begin{array}{c}\text { HERL } \\
(\boldsymbol{n}=\mathbf{1 4})\end{array}$ \\
\hline Contact Angle $\left(^{\circ}\right)$ & $83.4 \pm 5.05$ & $71.5 \pm 4.18$ & $87.0 \pm 5.28^{*}$ \\
Average Velocity $(\mathrm{m} / \mathrm{s})$ & $1.02 \pm 0.06$ & $0.95 \pm 0.05$ & $1.09 \pm 0.06$ \\
Cadence (stroke/s) & $1.03 \pm 0.07$ & $0.98 \pm 0.06$ & $1.04 \pm 0.08$ \\
Peak Force $(\mathrm{N})$ & $60.3 \pm 5.07$ & $48.7 \pm 4.20$ & $69.7 \pm 5.30^{\dagger}$ \\
Average Force $(\mathrm{N})$ & $35.5 \pm 2.68$ & $29.0 \pm 2.22$ & $39.4 \pm 2.80^{\dagger}$ \\
Peak Moment $(\mathrm{N} \bullet \mathrm{m})$ & $12.6 \pm 0.93$ & $10.5 \pm 0.77$ & $15.5 \pm 0.97^{\ddagger}$ \\
Average Moment $(\mathrm{N} \bullet \mathrm{m})$ & $6.82 \pm 0.46$ & $5.60 \pm 0.39$ & $8.01 \pm 0.49^{\ddagger}$ \\
\hline
\end{tabular}

${ }^{*}$ HERL marginally significantly different from UW $(p=0.07)$.

${ }^{\dagger}$ HERL significantly different from UW $(p<0.05)$.

${ }^{\ddagger}$ HERL significantly different from UW $(p<0.005)$.

HERL = Human Engineering Research Laboratories, KMRREC = Kessler Medical Rehabilitation Research and Education Corporation, UW = University of Washington.

Table 3.

Constant speed trial at $1.8 \mathrm{~m} / \mathrm{s}$ : Temporal and kinetic data (mean \pm standard error) for each site after controlling for sex, years postinjury, and weight.

\begin{tabular}{lccc}
\hline \multicolumn{1}{c}{ Variable } & $\begin{array}{c}\text { KMRREC } \\
(\boldsymbol{n}=\mathbf{1 4})\end{array}$ & $\begin{array}{c}\text { UW } \\
(\boldsymbol{n}=\mathbf{1 4})\end{array}$ & $\begin{array}{c}\text { HERL } \\
(\boldsymbol{n}=\mathbf{1 4})\end{array}$ \\
\hline Contact Angle $\left(^{\circ}\right)$ & $85.9 \pm 3.94$ & $83.4 \pm 3.34$ & $92.8 \pm 4.08$ \\
Average Velocity $(\mathrm{m} / \mathrm{s})$ & $1.74 \pm 0.06$ & $1.79 \pm 0.05$ & $1.73 \pm 0.06$ \\
Cadence $($ stroke/s) & $1.26 \pm 0.09$ & $1.25 \pm 0.08$ & $1.27 \pm 0.10$ \\
Peak Force $(\mathrm{N})$ & $82.1 \pm 7.35$ & $79.5 \pm 6.09$ & $96.3 \pm 7.68$ \\
Average Force $(\mathrm{N})$ & $44.5 \pm 4.00$ & $40.9 \pm 3.32$ & $50.2 \pm 4.18$ \\
Peak Moment $(\mathrm{N} \bullet \mathrm{m})$ & $16.9 \pm 1.35$ & $15.7 \pm 1.12$ & $20.0 \pm 1.41^{*}$ \\
Average Moment $(\mathrm{N} \bullet \mathrm{m})$ & $8.35 \pm 0.73$ & $7.12 \pm 0.60$ & $9.42 \pm 0.76^{*}$
\end{tabular}

${ }^{*}$ HERL marginally significantly different from UW $(p=0.06)$.

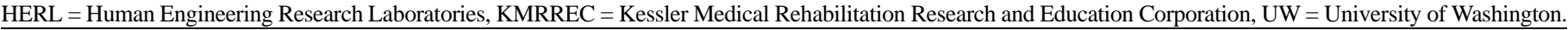

\section{Acceleration (First Stroke)}

Average velocity of the first stroke was significantly different between sites, with UW subjects starting out slower than subjects at the other two sites (Table 4). Because propulsion speed can influence the other propulsion variables, the ANCOVA was conducted, controlling for the average velocity of the first stroke in addition to sex, years postinjury, and body weight. No differences were found in the forces and moments for the first stroke among the three sites.

\section{Coastdown}

The ANOVA revealed a significant difference between sites, with UW subjects showing a slower coastdown 
deceleration compared with KMRREC and HERL subjects (Table 5). Site differences were also found for initial velocity at the start of coastdown, with UW subjects pushing at a slower speed compared with KMRREC subjects. Mean \pm standard deviation (SD) coastdown times from $1 \mathrm{~m} / \mathrm{s}$ were $4.18 \pm 1.0 \mathrm{~s}$ (UW), $2.66 \pm 0.62 \mathrm{~s}$ (KMRREC), and $2.49 \pm$ $0.74 \mathrm{~s}$ (HERL) $(p<0.001)$. UW was significantly different from both HERL and KMRREC $(p<0.001)$.

The slower rate of coastdown for subjects at UW implies that rolling resistance was less for UW compared with the other sites. Less rolling resistance would explain the lower propulsive torque and force needed by UW subjects to push at the two steady state speeds. A correction method was developed that used the difference in the rolling resistance between sites to offset the force and moment data at the UW site. The correction method is described in more detail in the Appendix (available online only at http://www.rehab.research.va.gov/). The same statistical model used in the original data set was used to test for differences in the modified data set. Weight was removed as a covariate since the correction method accounted for body weight in the rolling resist- ance calculations. The next section shows the results after applying the correction method to this data set.

\section{Correction}

\section{Step 1: Inertia}

The mean \pm SD wheel/roller inertia for each site were $3.80 \pm 0.63 \mathrm{~kg}-\mathrm{m}^{2} / \mathrm{s}(\mathrm{KMRREC}), 3.17 \pm 0.40 \mathrm{~kg}-\mathrm{m}^{2} / \mathrm{s}$ (UW), and $3.25 \pm 0.39 \mathrm{~kg}-\mathrm{m}^{2} / \mathrm{s}$ (HERL). These values were close to the values reported in a prior study for the combined SmartWheel (earlier model) and wheelchair dynamometer (on which the dynamometers in this study were modeled) [15]: $3.56 \pm 0.01 \mathrm{~kg}-\mathrm{m}^{2} / \mathrm{s}$ (left side) and $3.47 \pm 0.01 \mathrm{~kg}-\mathrm{m}^{2} / \mathrm{s}$ (right side).

\section{Step 2: Rolling Resistance}

Rolling resistance was highest at KMRREC, followed by HERL, and lastly UW (Table 6).

The coefficients of friction for each site were 0.06 (KMRREC), 0.04 (UW), and 0.06 (HERL) for the $0.9 \mathrm{~m} / \mathrm{s}$ trial and 0.05 (KMRREC), 0.03 (UW), and 0.05 (HERL) for the $1.8 \mathrm{~m} / \mathrm{s}$ trial.

Table 4.

First stroke of acceleration-brake-coastdown trial: Temporal and kinetic data (mean \pm standard error) for each site after controlling for sex, years postinjury, weight, and average velocity.

\begin{tabular}{lccc}
\hline \multicolumn{1}{c}{ Variable } & $\begin{array}{c}\text { KMRREC } \\
(\boldsymbol{n}=\mathbf{~ 1 4 )}\end{array}$ & $\begin{array}{c}\text { UW } \\
(\boldsymbol{n}=\mathbf{1 4})\end{array}$ & $\begin{array}{c}\text { HERL } \\
(\boldsymbol{n}=\mathbf{1 4})\end{array}$ \\
\hline Contact Angle $\left(^{\circ}\right)$ & $82.4 \pm 5.90$ & $70.0 \pm 5.81$ & $80.7 \pm 5.97$ \\
Average Velocity $(\mathrm{m} / \mathrm{s})$ & $0.82 \pm 0.05^{*}$ & $0.56 \pm 0.04$ & $0.73 \pm 0.05^{\dagger}$ \\
Cadence $($ stroke/s) & $1.01 \pm 0.09$ & $1.11 \pm 0.08$ & $1.10 \pm 0.09$ \\
Peak Force $(\mathrm{N})$ & $141.9 \pm 10.85$ & $110.6 \pm 10.70$ & $115.3 \pm 10.99$ \\
Average Force $(\mathrm{N})$ & $78.9 \pm 6.49$ & $65.6 \pm 6.40$ & $70.6 \pm 6.57$ \\
Peak Moment $(\mathrm{N} \bullet \mathrm{m})$ & $32.9 \pm 2.69$ & $26.4 \pm 2.66$ & $27.7 \pm 2.72$ \\
Average Moment $(\mathrm{N} \cdot \mathrm{m})$ & $19.5 \pm 1.69$ & $15.4 \pm 1.67$ & $17.1 \pm 1.71$ \\
\hline
\end{tabular}

${ }^{*}$ KMRREC significantly different from UW $(p<0.001)$.

${ }^{\dagger}$ HERL significantly different from UW $(p=0.0497)$.

HERL = Human Engineering Research Laboratories, KMRREC $=$ Kessler Medical Rehabilitation Research and Education Corporation, UW $=$ University of Washington.

Table 5.

Curve-fit model constants for coastdown for each site, $d=1 / 2 a t^{2}+v t+d_{0}$. Data presented as mean \pm standard deviation.

\begin{tabular}{ccccc}
\hline Constant & $\begin{array}{c}\text { KMRREC } \\
(\boldsymbol{n}=\mathbf{1 4})\end{array}$ & $\begin{array}{c}\text { UW } \\
(\boldsymbol{n}=\mathbf{1 3})\end{array}$ & $\begin{array}{c}\text { HERL } \\
(\boldsymbol{n}=\mathbf{1 4})\end{array}$ & Significance \\
\hline$a$ & $-0.23 \pm 0.06$ & $-0.14 \pm 0.03^{*}$ & $-0.22 \pm 0.04$ & $<0.001$ \\
$v$ & $2.60 \pm 0.43$ & $1.98 \pm 0.45^{\dagger}$ & $2.21 \pm 0.53$ & 0.005 \\
$d_{0}$ & $0.04 \pm 0.03$ & $0.05 \pm 0.03$ & $0.04 \pm 0.03$ & 0.469 \\
\hline
\end{tabular}

*UW significantly different from KMRREC $(p<0.001)$ and HERL $(p<0.001)$.

${ }^{\dagger}$ UW significantly different from KMRREC $(p=0.005)$.

$a=$ acceleration $\left(\mathrm{m} / \mathrm{s}^{2}\right), d=$ distance traveled $(\mathrm{m}), d_{0}=$ initial distance $(\mathrm{m})$, HERL $=$ Human Engineering Research Laboratories, KMRREC $=$ Kessler Medical Rehabilitation Research and Education Corporation, $t=$ time (s), UW = University of Washington, $v=$ initial velocity (m/s). 


\section{Step 3: Site Normalization of Pushrim Kinetic Data}

Tables 7 and $\mathbf{8}$ show the site-normalized forces and moments for KMRREC for the $0.9 \mathrm{~m} / \mathrm{s}$ and $1.8 \mathrm{~m} / \mathrm{s}$ propulsion trials, respectively. After normalizing the data, the new mean UW forces and moments were increased on average by about $4 \mathrm{~N}$ and $1 \mathrm{~N} \bullet \mathrm{m}$, respectively (comparing Tables 2 and $\mathbf{3}$ with Tables $\mathbf{7}$ and $\mathbf{8}$ ). KMRREC's new mean forces ranged from $<1 \mathrm{~N}$ to $5 \mathrm{~N}$ depending on the speed and $<1 \mathrm{~N} \bullet \mathrm{m}$ for the moments after the normalization. None of the force and moment variables were statistically different between sites with the ANCOVA.

\section{DISCUSSION}

A multisite study presents a unique opportunity to study the propulsion techniques of a large group of manual wheelchair users from different geographical regions. However, a study of this nature requires precautions that ensure uniformity in protocol execution, equipment, and instrumentation. While each site followed the same pro- tocol and used the same type of dynamometer and instrumented wheel, UW was found initially to be different from HERL with respect to steady state propulsion force and torque. We first considered differences in the subject characteristics as a potential source for the differences in force and torque. We found that subjects at UW were similar in age, weight, and injury level to subjects at HERL and KMRREC. Although not significant, UW had more women in its sample, and both UW and KMRREC differed statistically from HERL on years post-SCI. Also, body weight is related to the force required to propel [5]. Thus, we decided to control for three variables in our statistical model: body weight, sex, and years post-SCI. Controlling for these subject differences, we still found that force and torque were different at UW compared with HERL. This finding led us to analyze the coastdown portion of the acceleration trial. From the analysis of coastdown, rolling resistance appeared to be the likely source for the differences in force and torque because UW subjects had the slowest initial velocities at the

Table 6.

Rolling resistance (mean \pm standard deviation) for each site for 0.9 and $1.8 \mathrm{~m} / \mathrm{s}$ trials.

\begin{tabular}{cccc}
\hline Rolling Resistance (N) & $\begin{array}{c}\text { KMRREC } \\
(\boldsymbol{n}=\mathbf{1 4})\end{array}$ & $\begin{array}{c}\text { UW } \\
(\boldsymbol{n}=\mathbf{1 3})\end{array}$ & $\begin{array}{c}\text { HERL } \\
(\boldsymbol{n}=\mathbf{1 4})\end{array}$ \\
\hline $0.9 \mathrm{~m} / \mathrm{s}$ & $17.39 \pm 6.67$ & $9.27 \pm 3.28$ & $14.28 \pm 3.85$ \\
$1.8 \mathrm{~m} / \mathrm{s}$ & $14.47 \pm 5.89$ & $8.85 \pm 4.04$ & $13.53 \pm 3.68$ \\
\hline HERL = Human Engineering Research Laboratories, KMRREC = Kessler Medical Rehabilitation Research and Education Corporation, UW = University of Washington.
\end{tabular}

Table 7.

Constant speed trial at $0.9 \mathrm{~m} / \mathrm{s}$ : Average and peak force and moment variables (mean \pm standard error) after site-normalizing UW and KMRREC data.

\begin{tabular}{lccc}
\hline \multicolumn{1}{c}{ Variable } & $\begin{array}{c}\text { KMRREC } \\
(\boldsymbol{n}=\mathbf{1 4})\end{array}$ & $\begin{array}{c}\text { UW } \\
(\boldsymbol{n}=\mathbf{1 3})\end{array}$ & $\begin{array}{c}\text { HERL } \\
(\boldsymbol{n}=\mathbf{1 4})\end{array}$ \\
\hline Peak Force $(\mathrm{N})$ & $61.3 \pm 5.60$ & $52.9 \pm 4.90$ & $64.9 \pm 5.86$ \\
Average Force $(\mathrm{N})$ & $35.3 \pm 3.14$ & $33.5 \pm 2.75$ & $36.1 \pm 3.29$ \\
Peak Moment $(\mathrm{N} \bullet \mathrm{m})$ & $12.7 \pm 1.13$ & $11.7 \pm 0.99$ & $14.4 \pm 1.18$ \\
Average Moment $(\mathrm{N} \bullet \mathrm{m})$ & $6.60 \pm 0.61$ & $6.91 \pm 0.54$ & $7.32 \pm 0.64$ \\
\hline HERL = Human Engineering Research Laboratories, KMRREC = Kessler Medical Rehabilitation Research and Education Corporation, UW = University of Washington.
\end{tabular}

Table 8.

Constant speed trial at $1.8 \mathrm{~m} / \mathrm{s}$ : Average and peak force and moment data (mean \pm standard error) after site-normalizing UW and KMRREC data.

\begin{tabular}{lccc}
\hline \multicolumn{1}{c}{ Variable } & $\begin{array}{c}\text { KMRREC } \\
(\boldsymbol{n}=\mathbf{1 4})\end{array}$ & $\begin{array}{c}\text { UW } \\
(\boldsymbol{n}=\mathbf{1 3})\end{array}$ & $\begin{array}{c}\text { HERL } \\
(\boldsymbol{n}=\mathbf{1 4})\end{array}$ \\
\hline Peak Force $(\mathrm{N})$ & $87.5 \pm 8.03$ & $83.8 \pm 7.02$ & $89.4 \pm 8.38$ \\
Average Force $(\mathrm{N})$ & $47.4 \pm 4.29$ & $45.2 \pm 3.75$ & $47.0 \pm 4.48$ \\
Peak Moment $(\mathrm{N} \bullet \mathrm{m})$ & $18.1 \pm 1.51$ & $17.1 \pm 1.32$ & $18.6 \pm 1.57$ \\
Average Moment $(\mathrm{N} \bullet \mathrm{m})$ & $9.17 \pm 0.85$ & $8.36 \pm 0.74$ & $8.67 \pm 0.88$ \\
\hline HERL = Human Engineering Research Laboratories, KMRREC = Kessler Medical Rehabilitation Research and Education Corporation, UW = University of Washington.
\end{tabular}


beginning of coastdown, yet we found that they also had the lowest decelerations and longer coastdown times than subjects at the other two sites.

By solving the quadratic equation for parabolic motion to determine the subject/wheelchair deceleration, we assumed that the deceleration of the subject/wheelchair was constant. To determine whether this assumption was valid, we plotted the subjects' velocity versus time curves during the coastdown portion of the trial. Given that the relationship approximated linear, our assumption appeared to be confirmed. In addition, we found that the predicted distance curve based on the model equation closely matched the measured distance curve.

Several non-subject-related factors could be responsible for the differences in forces and moments observed across sites. These factors include the internal rolling resistance of the SmartWheel and the wheelchair dynamometer, the calibration of the SmartWheel and the dynamometer, and wheelchair setup. The SmartWheel is designed to attach by way of a customized axle to any wheelchair with quick-release wheels. Tightening down a screw that is threaded through the custom axle secures the wheel to the wheelchair. Slight variations in the machining and wear of the axle may affect the rolling resistance of the SmartWheel; however, the effects of this would likely be too small to result in the site differences in forces and moments found.

The SmartWheel readings could also be responsible for the differences in forces and moments between sites. If the calibration of the SmartWheel were off, then the calculations for the propulsion forces and moments would be affected. A formal calibration of the SmartWheel is performed by the manufacturer before the wheel is sent out; however, to ensure that the wheel is measuring properly, every site (independently) conducts a calibration check every 6 months. We chose this time frame for checking the calibration because our past and continued experiences with the SmartWheel have found that the constants remain stable unless a sensor is damaged, in which case it is immediately detectable when viewing the data and the wheel is repaired and then recalibrated. Each site's SmartWheel calibration checks indicated that the constants remained stable between checks, and therefore the SmartWheel readings are an unlikely source for the differences in the forces and moments between sites.

The most likely source for the differences between sites is the rolling resistance inherent in the dynamometer. Sites routinely perform a maintenance procedure that entails lubrication of the bearings; however, the amount of lubrication that actually surrounds the bearings is impossible to assess and therefore could vary across sites and cause rolling resistance to differ across sites. Tolerances in the bearings and pillow blocks; wear; or small changes in alignment of the frame, pillow blocks, or rollers could also be partly responsible for the site-to-site differences.

To check the dynamometer performance across sites, we routinely conduct unloaded coastdown tests. We observed, however, that the unloaded coastdowns did not seem to correlate with the results of the subject (loaded) testing in this study. For instance, KMRREC's unloaded coastdown times were almost twice as long as the coastdown times at UW and HERL, and UW and HERL were very similar to each other. Conducting a loaded calibration test across sites is difficult because it would require the same load distribution over the rollers (e.g., same person, same wheelchair, same frame configuration, tiedown). Since one of our HERL investigators is a wheelchair user, we were able to conduct a coastdown test at UW during our annual in-person meeting and compare the results with HERL. This investigator used the same wheelchair and setup and did not gain or lose weight between tests. His deceleration, as determined with the Equation (p. 452), was $0.19 \mathrm{~m} / \mathrm{s}^{2}$ at HERL and $0.14 \mathrm{~m} / \mathrm{s}^{2}$ at UW; his time to coastdown from $1 \mathrm{~m} / \mathrm{s}$ was $3.1 \mathrm{~s}$ at HERL and $4.1 \mathrm{~s}$ at UW. These results concur with the findings of our study that UW subjects face less resistance than HERL subjects and that the dynamometer is likely the primary source for the site differences in the propulsion forces and moments.

The distribution of the person's weight in the wheelchair can significantly impact rolling resistance [16]. Weight distribution over the dynamometer depends on rear wheel location and the method of tie-down. Data on weight distribution in the wheelchair were only available for HERL subjects and thus could not be compared with the other sites. While different tie-down fixtures were used to secure the wheelchair to the dynamometer, they were all designed to maintain the subject/wheelchair's weight distribution over the front and rear tires.

The differences in pushrim kinetics at the slow speed, however, did not all carry over to the faster speed. Thus, the difference in rolling resistance is probably not due to wheelchair set up, tie-down methods, or the SmartWheel but, again, likely related to dynamometer properties. This conclusion is why we chose to approach the problem by using a mechanical model to estimate 
rolling resistance and site-normalize the data. Because rolling resistance appeared to be velocity dependent (e.g., greater differences in propulsion forces and moments were found at the slower versus the faster steady state speed), we applied our normalization technique to each speed trial independently. For instance, we calculated rolling resistance for the slow speed trial and used it to offset the forces and moments at the slow speed. This solution to normalize the site data may allow other research groups collecting data on wheelchair propulsion to compare and combine their data. Part of the correction is based on group results; therefore, as people are added to the study, reassessing the conditions of the coastdown and modifying accordingly the methods used to correct the data is important.

Other wheelchair propulsion studies have used either a wheelchair ergometer [17-19], roller system [10], or treadmill [20-21] to simulate wheelchair motion. Regardless of which device one chooses, the challenge of ensuring that equipment is operating similarly remains. De Groot et al., in a multisite study involving eight rehabilitation centers, discovered that the measured propulsion power output of seven identical motor-driven treadmills and one specially built motor-driven treadmill varied considerably, and they investigated the reasons why [22]. They found that the source of the difference in power output between sites was not calibration, wheelchair occupant, or experimenter errors but rather differences in treadmill characteristics. For instance, the actual treadmill slopes did not match the programmed slopes and the difference between the two was not consistent across sites. They also found that measured velocities differed from the velocities on the treadmill display and that increasing the wheelchair load affected the measured velocity. Furthermore, wheelchair factors influenced the measured drag forces [22]. Overground propulsion eliminates some of the concerns associated with wheelchair simulators, but standardizing the experimental setup is still important in a multisite study (e.g., ensuring that propulsion surfaces and slopes are identical and that devices used to record propulsion speed, force, etc., are accurately calibrated). In addition, overground propulsion makes control of velocity and kinematic data collection over more than a couple strokes very challenging.

This study focused on kinetic differences in propulsion techniques across sites. In addition to collecting kinetic data on propulsion technique, each site also collected full upper-body kinematic data for each trial. A future analysis will look at kinematic differences (e.g., joint angles, range of motion, propulsion patterns) across sites. We chose to start with investigating the total force and wheel moment and some basic temporal variables to obtain a general sense of whether subjects at each site were propelling with similar kinetics. Eventually, we will combine the data and apply an inverse dynamics model to investigate the relationship between joint biomechanics and upper-limb pain and injury.

\section{CONCLUSIONS}

Conducting a large scale multisite, multicenter study presents investigators with the challenge of ensuring that research protocols and instrumentation are uniform across sites so that the data collected can be pooled. When comparing propulsion variables across three sites, we discovered that subjects at one site pushed with lower force and torque compared with the other sites. Analysis of a coastdown trial indicated that this difference is likely related to the lower rolling resistance inherit in the wheelchair dynamometer. Using a mechanical model, we were able to correct for the kinetic differences between sites so that the data could be combined and used in future analyses.

\section{ACKNOWLEDGMENTS}

A special thanks to Dianxu Ren, $\mathrm{PhD}$, who assisted with statistical analysis, and to Ian Rice, who permitted the use of his photograph.

Carmen P. DiGiovine, PhD, RET, is now with 6 Degrees of Freedom, LLC, Wheaton, Illinois.

This material was based on work supported by the National Institute on Disability and Rehabilitation Research (NIDRR) (grant NIDRR H133A011107), the Department of Veterans Affairs (Rehabilitation Research and Development Service project B3057R), and the University of Pittsburgh Model Center on Spinal Cord Injury (grant NIDRR H133N000019).

The authors have declared that no competing interests exist.

\section{REFERENCES}

1. Sie IH, Waters RL, Adkins RH, Gellman H. Upper extremity pain in the postrehabilitation spinal cord injured patient. Arch Phys Med Rehabil. 1992;73(1):44-48. [PMID: 1729973] 
2. Pentland WE, Twomey LT. The weight-bearing upper extremity in women with long term paraplegia. Paraplegia. 1991;29(8):521-30. [PMID: 1775358]

3. Nichols PJ, Norman PA, Ennis JR. Wheelchair user's shoulder? Shoulder pain in patients with spinal cord lesions. Scand J Rehabil Med. 1979;11(1):29-32. [PMID: 419395$]$

4. Nyland J, Quigley P, Huang C, Lloyd J, Harrow J, Nelson A. Preserving transfer independence among individuals with spinal cord injury. Spinal Cord. 2000;38(11):649-57. [PMID: 11114770]

5. Boninger ML, Cooper RA, Baldwin MA, Shimada SD, Koontz AM. Wheelchair pushrim kinetics: Body weight and median nerve function. Arch Phys Med Rehabil. 1999; 80(8):910-15. [PMID: 10453767]

6. Boninger ML, Impink BG, Cooper RA, Koontz AM. Relation between median and ulnar nerve function and wrist kinematics during wheelchair propulsion. Arch Phys Med Rehabil. 2004;85(7):1141-45. [PMID: 15241765]

7. Boninger ML, Koontz AM, Sisto SA, Dyson-Hudson TA, Chang M, Price R, Cooper RA. Pushrim biomechanics and injury prevention in spinal cord injury: Recommendations based on CULP-SCI investigations. J Rehabil Res Dev. 2005; 42(3 Suppl 1):9-19. [PMID: 16195959]

8. Mercer JL, Boninger ML, Koontz AM, Ren D, DysonHudson TA, Cooper R. Shoulder joint kinetics and pathology in manual wheelchair users. Clin Biomech (Bristol, Avon). 2006;21(8):781-89. [PMID: 16808992]

9. Van Drongelen S, Van der Woude LH, Janssen TW, Angenot EL, Chadwick EK, Veeger DH. Mechanical load on the upper extremity during wheelchair activities. Arch Phys Med Rehabil. 2005;86(6):1214-20. [PMID: 15954062]

10. Rodgers MM, Gayle GW, Figoni SF, Kobayashi M, Lieh J, Glaser RM. Biomechanics of wheelchair propulsion during fatigue. Arch Phys Med Rehabil. 1994;75(1):85-93. [PMID: 8291970]

11. Mulroy SJ, Newsam CJ, Gutierrez DD, Requejo P, Gronley JK, Haubert LL, Perry J. Effect of fore-aft seat position on shoulder demands during wheelchair propulsion: Part 1. A kinetic analysis. J Spinal Cord Med. 2005;28(3):214-21. [PMID: 16048139]

12. Sabick MB, Zhao KD, An KN. A comparison of methods to compute the point of force application in handrim wheel- chair propulsion: A technical note. J Rehabil Res Dev. 2001; 38(1):57-68. [PMID: 11322471]

13. VanSickle DP, Cooper RA, Robertson RN. SmartWheel: Development of a digital force and moment sensing pushrim. Proceedings of the 18th Annual RESNA Conference; 1995 June 9-14; Vancouver, Canada. Washington (DC): RESNA Press; 1995. p. 352-54.

14. Cooper RA, Robertson RN, VanSickle DP, Boninger ML, Shimada SD. Methods for determining three-dimensional wheelchair pushrim forces and moments: A technical note. J Rehabil Res Dev. 1997;34(2):162-70. [PMID: 9108343]

15. DiGiovine CP, Cooper RA, Boninger ML. Dynamic calibration of a wheelchair dynamometer. J Rehabil Res Dev. 2001;38(1):41-55. [PMID: 11322470]

16. Boninger ML, Baldwin M, Cooper RA, Koontz AM, Chan L. Manual wheelchair pushrim biomechanics and axle position. Arch Phys Med Rehabil. 2000;81(5):608-13. [PMID: 10807100]

17. Dallmeijer AJ, Van der Woude LH, Veeger HE, Hollander AP. Effectiveness of force application in manual wheelchair propulsion in persons with spinal cord injuries. Am J Phys Med Rehabil. 1998;77(3):213-21. [PMID: 9635556$]$

18. Veeger HE, Van der Woude LH, Rozendal RH. A computerized wheelchair ergometer. Results of a comparison study. Scand J Rehabil Med. 1992;24(1):17-23. [PMID: 1604258]

19. Brown DD, Knowlton RG, Hamill J, Schneider TL, Hetzler RK. Physiological and biomechanical differences between wheelchair-dependent and able-bodied subjects during wheelchair ergometry. Eur J Appl Physiol Occup Physiol. 1990; 60(3):179-82. [PMID: 2347318]

20. Sanderson DJ, Sommer HJ 3rd. Kinematic features of wheelchair propulsion. J Biomech. 1985;18(6):423-29. [PMID: 4030799]

21. Richter WM, Rodriquez R, Woods KR, Axelson PW. Consequences of a cross slope on wheelchair handrim biomechanics. Arch Phys Med Rehabil. 2007;88(1)76-80. [PMID: 17207679]

22. De Groot S, Zuidgeest M, Van der Woude LH. Standardization of measuring power output during wheelchair propulsion on a treadmill. Pitfalls in a multi-center study. Med Eng Phys. 2006;28(6):604-12. [PMID: 16300988]

Submitted for publication May 23, 2006. Accepted in revised form January 3, 2007. 